\title{
Impacto de la Disciplina Gestión de Personas en los Cursos Universitarios de Tecnología en Seguridad de la Información
}

\author{
Marcio Romero y Renato J. Sassi \\ Ingeniería de Producción, Universidad Nove de Julho, Rua Francisco Matarazzo no 612 , CEP: \\ 05001-000, São Paulo/SP-Brasil. (e-mail: sassi@uninove.br y mromero@uninove.br)
}

Recibido Sep. 26, 2011; Aceptado Nov. 22, 2011; Versión final recibida Dic. 27, 2011

\begin{abstract}
Resumen
Se ha estudiado el impacto de la disciplina de Gestión de Personas en los estudiantes de los cursos de Tecnología en Seguridad de la Información de una Universidad privada en la ciudad de São Paulo en Brasil. Muchos profesionales en el área de tecnología de la información hacen sus carreras a base de cursos y certificaciones técnicas que no consideran que la Gestión de Personas sea un factor clave para obtener buenos resultados en su trabajo profesional. El estudio muestra la importancia de esta disciplina de gestión, la que no solo debe ser tratada de una manera puramente teórica. Los estudios de caso son fundamentales para una mejor comprensión y aplicación del conocimiento adquirido.
\end{abstract}

Palabras clave: seguridad de la información, gestión de personas, cursos universitarios, casos de estudio

\section{Impact of the People Management Discipline on Technology on Information Security Courses for Undergraduate Students}

\begin{abstract}
The impact of the discipline People Management on students of Technology on Information Security of a private University in the city of São Paulo in Brazil has been studied. Many professionals in the information technology area take theoretical courses and get technical certifications that do not consider that People Management is a key factor to achieve good results in their professional careers. This study demonstrates the importance of the discipline People Management and that it should not be only theoretically covered. Study cases are of fundamental importance for better understanding and application of the knowledge acquired.
\end{abstract}

Keywords: information security, people management, undergraduate courses, study cases 


\section{INTRODUCCIÓN}

En el escenario actual, donde las empresas entran en un mercado competitivo y globalizado, maximizar los resultados sin aumentar los costos y mantenerse al día con la calidad es una necesidad. En este contexto, las Tecnologías de la Información (TI) desempeñan un papel clave para permitirle a las empresas optimizar sus recursos y acceso a la información, lo que le permitirá a las mismas tomar decisiones asertivas y así potenciar sus negocios.

La Tecnología en Seguridad de la Información (SI) está relacionada con la protección de un conjunto de datos con el fin de preservar el valor que estos tienen para la organización. Sus características básicas son los atributos de confidencialidad, integridad y autenticidad. Por lo tanto, las decisiones en el SI, que antes podían considerarse de forma aislada en el área de TI, ahora debe ser consideradas en el contexto del negocio.

En los últimos años, como resultado de cambios en el entorno corporativo, el perfil profesional de la SI está evolucionando hacia un nuevo modelo, que requiere una interfaz con otros equipos y profesionales de negocios y también tener en cuenta la importancia del factor humano. Todos los profesionales, independientemente del área en que trabajen, deberían tener, al menos, algún conocimiento de Gestión de Personas, que le permita mejorar su relación con los recursos humanos, permitiéndole, no solamente desarrollar sus capacidades, sino también superar sus propios límites.

El líder perfecto en el área de $\mathrm{TI}$, o al menos, una descripción de aquel que se relaciona de la mejor manera con sus compañeros, poco importa la apariencia, lo más relevante, según los analistas, vendedores, ejecutivos y headhunters, son características como la eficiencia y la veracidad en la comunicación, y la forma en la que el profesional se relacione con la gente y como organiza su tiempo (Dalmazo, 2007).

La Gestión de Personas es el conjunto de decisiones integradas sobre las relaciones laborales que influyen en la eficacia de los empleados y organizaciones. Por lo tanto, todos los gerentes son, en cierto sentido, gerentes de personas, ya que todos ellos están involucrados en actividades tales como reclutamiento, entrevistas, selección y capacitación (Chiavenato, 2009).

Es evidente que muchos profesionales de TI hacen sus carreras a base de cursos y certificaciones técnicas, pero muchos olvidan que la Gestión de Personas es parte de su vida cotidiana, sobre todo cuando se tiene un rol de liderazgo profesional y la necesidad de ver una perspectiva empresarial más allá del área de TI.

Con base en estas consideraciones, es evidente que una manera de trabajar con temas de Gestión de Personas es tratarlos en las disciplinas académicas, lo que anima a los estudiantes, de Tecnología en Seguridad de la Información, sobre la manera de aplicar estos conceptos en su ambiente de trabajo, porque se trata de diversos temas que ayudan a los profesionales en las áreas de SI, a mejorar y aumentar su desempeño profesional cuando se trata de temas relacionados con asuntos relacionados con las personas.

El curso de Tecnología en Seguridad de la Información se centra en la preparación de profesionales para asegurar la integridad y la protección de las organizaciones de información, los protege de accesos no autorizados.

Así, dentro de los principios de confidencialidad, integridad y disponibilidad, el trabajador gestiona, administra, gestiona y configura los ambientes empresariales con los requisitos de seguridad. Realiza análisis de riesgo, gestiona los sistemas de información, diseña y administra las redes de computadoras seguras, auditorías, planes de contingencia y recuperación de información en caso de accidentes. Opera en los aspectos físicos y lógicos, el control de los niveles de acceso a los sistemas operativos, bases de datos y redes informáticas (4hd, 2010). 
Sin embargo, gestionar el desarrollo de la disciplina Gestión de Personas en estos cursos se convierte en un gran desafío para los profesores, ya que los estudiantes están más interesados en las disciplinas técnicas.

El plan de estudios del curso de gestión de personas tienen un enfoque conceptual, donde los temas se tratan desde una perspectiva más filosófica que a través de la aplicación práctica.

Galve-Górriz y Castelm (2010) argumentan que la fuerte relación entre los aspectos de la tecnología diversa y de recursos humanos, elementos clave en la competitividad de la empresa, son complementarios. Teniendo en cuenta estos resultados, es evidente que requiere la gestión de los cambios tecnológicos correspondientes as las políticas de recursos humanos.

De este argumento surge la siguiente pregunta: ¿cuál es el impacto de la disciplina de Gestión de Personas en los cursos de graduación de Tecnología en Seguridad de la Información?

Encontrar la respuesta a esta pregunta, con la participación del estudiante, es el principal objetivo de este trabajo. Para apoyar este estudio, se realizó una encuesta con los grupos del segundo, tercero y cuarto semestre de los cursos de Tecnología en Seguridad de Información en una universidad privada en San Pablo, sin embargo, la disciplina de la Gestión de Personas sólo se incluye en el cuarto semestre del curso en cuestión.

El objetivo de este artículo es poner de relieve la importancia de esta disciplina en la Gestión de Personas para los estudiantes en el curso y el objetivo específico es evaluar el nivel de comprensión, el interés por la disciplina y la credibilidad de la misma, con el objetivo de entender cómo esta disciplina contribuirá a su participación en el mercado profesional.

En este artículo se presentan los resultados de esta investigación, en los que se expresará la visión de la disciplina de Gestión de Personas. Analizaremos también algunos aspectos de como abordar la disciplina, así como la aplicabilidad de la misma en los negocios.

\section{REVISIÓN DE LA LITERATURA}

La preocupación con la Gestión de Personas se comenzó en la Revolución Industrial, debido a presiones sindicales y al recelo frente a la amenaza que estas organizaciones laborales representaban para las organizaciones de Inglaterra y los Estados Unidos. Su estructuración, sin embargo, tuvo lugar en el siglo XX con el advenimiento de la Escuela de Administración Científica. Esta escuela coloca a la gestión de recursos humanos de las organizaciones como responsables por proporcionar una fuerza de trabajo efectivo a las organizaciones (Dutra, 2004).

La mayoría de las empresas se han dado cuenta de que tener un profesional técnico altamente calificado, no es suficiente. Si este no consigue comunicarse con el resto del personal de otras áreas de la organización o, incluso, de su propio equipo, es evidente que este profesional no traerá buenos resultados para la organización.

En el área de la SI, con el tema de Gestión de Personas, no podía ser diferente porque esta es un área que se relaciona de manera esencial con las otras áreas de la empresa. Realizar la administración de personal no es una tarea fácil, ya que además de conocimientos técnicos, es necesario tener un buen conocimiento de cómo tratar con la gente, no sólo de su equipo, sino también de los otros equipos, suministradores, socios y clientes.

El factor humano está tomando en un importante espacio dentro de la empresa y no podría ser diferente en el área de Seguridad de la Información, ya que la definición de Gestión de Personas se ve como un conjunto de políticas y prácticas de la organización dado para guiar el comportamiento humano y las relaciones interpersonales (Fleury, 2001).

Puesto que la disciplina de Gestión de Personas se ocupa de diversas cuestiones que afectan la carrera, como el tema del liderazgo, habilidades interpersonales, trabajo en equipo, comunicación, 
gestión de conflictos y los procesos de toma de decisiones para los grupos y sus opciones, y trata también de temas esenciales para un profesional que comienza su carrera, entonces permite la capacitación para alcanzar un lugar en el mercado de trabajo con conocimientos sistemáticos de gestión de personas y no solo con habilidades técnicas.

Neste contexto hay una necesidad de que el gerente de Seguridad de la Información motive al personal para que acepte los cambios implementados. Esto debe hacerse buscando el beneficio de la organización en su conjunto (Ruiz, 2007).

Rezende (2002) presenta sugerencias sobre el equipo de gestión durante la ejecución de los proyectos y lista las posibles características personales que distinguen un profesional de otro, haciéndolo más adecuado para ejecutar una actividad determinada, como la necesidad de un profesional con habilidades en la comunicación a cara a cara con el cliente y un experto en el área de desarrollo.

No hay una estrategia clara sobre la manera de instaurar un sistema que se ajuste a las necesidades de información de los usuarios. La tendencia de las instituciones de mucho estrés en las tecnologías, procesos internos y los factores institucionales (Allen, Wilson, 1997).

Organizaciones ven la necesidad de cuidar más acerca de las personas con la gestión de la información y la capacidad de innovar y renovar todo el tiempo, permaneciendo alerta para el medio ambiente interno y externo a sus límites. En esta era en que la sociedad se caracteriza por interactivo e interconectado en tiempo real, la información ha emergido como el activo clave para asegurar la supervivencia de las organizaciones (Starec et al., 2005).

Además de las organizaciones requieren de una evaluación sistemática, puede seguir el crecimiento de las personas que realizan sus funciones y al mismo tiempo, hacer una conexión con los principales objetivos de las unidades no sólo que las personas que están vinculadas, sino también con propósitos de la organización como un todo (Araujo, 2006).

A través de la literatura podemos entender que cambiar la forma de gestionar las personas dentro de una organización no es una tarea sencilla, se trata de un proceso a largo plazo ya que implica cambios reales en la cultura organizacional y requiere la participación activa de la alta administración y de todos los componentes de la organización.

Según Longo (2009), la gestión de la personas no puede ser vista de forma aislada de otros subsistemas de gestión que existen en cualquier organización. El vínculo común entre todos ellos es la estrategia. Los resultados dependen, en gran medida, del comportamiento de la gente en el trabajo. Si este comportamiento se ajusta adecuadamente a los objetivos de la empresa, las probabilidades de éxito son mayores.

Algunas de las principales cualidades que deben formar el perfil deseado en los profesionales de la SI son: actitud, ética, flexibilidad, liderazgo, dinamismo y buenas habilidades interpersonales, y estas habilidades se abordan en la disciplina de Administración de Personal.

Se puede notar una mejora en la estructura de SI, pero en la mayoría de las empresas (28\%) la tarea de cuidar de este departamento sigue perteneciendo al Gerente de TI / Redes. En algunos (16\%), el principal actor es el director de TI, y sólo en un número menor (13\%), la posición de Director de Seguridad existe (Security Review Journal, 2007).

Los estudiantes que se gradúan, tienen una gran preocupación para aprender todo acerca de las nuevas tecnologías y garantizar la seguridad de la información, pero todo este enfoque de aprendizaje es de carácter técnico, la percepción de gestión se aprende con el tiempo durante su experiencia de trabajo en las empresas.

La Gestión de Personas durante los cursos de graduación puede proporcionar un gran diferencial y preparar a los estudiantes para su entrada al mercado de trabajo. 
El entendimiento de que la disciplina de Gestión de Personas es un agente importante para la transformación de las carreras de los estudiantes será, ciertamente, un valor añadido y así, transformará la forma en la que estos estudiantes y futuros profesionales serán vistos y tratados por las organizaciones.

\section{METODOLOGÍA DE LA INVESTIGACIÓN}

La investigación se realizó con los estudiantes de Tecnología en Seguridad de la Información de una universidad privada de São Paulo.

Una encuesta puede ser considerada de carácter exploratorio, cuando se trata de revisión de la literatura, entrevistas con personas que tienen, o tener experiencia práctica con el problema investigado y analizado estudios de caso que fomenten la comprensión. La investigación exploratoria é dirigida a proporcionar una visión general de un hecho particular (Marconi, Lakatos, 2007).

Este estudio se basa en encuestas realizadas en 110 estudiantes (20\% de los estudiantes totales) a partir del segundo, tercero y cuarto semestre (durante la etapa final de cada semestre) de la licenciatura en curso de Tecnología en Seguridad de la Información, que ya han estudiado Gestión de Personas y otros que no la han estudiado aún, para que, de esa manera fuera posible analizar el perfil de estos estudiantes y su visión sobre la disciplina de Gestión de Personas, también entender qué grado de importancia le atribuyen a la cuestión de la Gestión de Personas en sus carreras.

El modelo utilizado para el desarrollo de esta investigación se compone de dos etapas:

Una para definir el perfil del estudiante, centrándose en las características del alumno, edad, sexo, el trabajo y en el área de especialización.

En la segunda parte se prestó atención a la disciplina de Gestión de Personas, con el fin de mostrar el grado de conocimiento y aplicación de la disciplina.

Para los grupos que ya han estudiado esta disciplina se utilizó un cuestionario diferente con un enfoque cualitativo, con el objetivo de identificar lo que se absorbe de este tema y si son capaces de aplicar los conocimientos adquiridos en su día a día.

Teniendo en cuenta el material seleccionado, se trató de observar los cambios realizados con la introducción de la disciplina, a través de cambio de actitudes en la empresa.

Una vez que La evaluación del aprendizaje consiste en algo más que asignar una calificación; además de medir el progreso del estudiante, muestra su potencial y limitaciones para avanzar en el conocimiento de la materia que se evalúa (Cano; Hernandez, 2011).

\section{RESULTADOS Y ANÁLISIS}

Los resultados de esta encuesta fueron tabulados, analizados y convergen de manera que podamos ratificar el estudio propuesto sobre la importancia de esta disciplina de los cursos de Tecnología de Gestión de Personas en la Seguridad de la Información.

Algunos factores que influyen son analizados en este estudio, tales como liderazgo, trabajo en equipo y la comprensión de los contenidos tratados en la disciplina y las posibles mejoras para hacerlo más dinámico y atractivo, ya que el perfil de los estudiantes del curso es de las disciplinas más técnicas y prácticas técnicas.

La investigación de base para este estudio se llevó a cabo con los grupos de Tecnología de la Información de Seguridad elegidos por la diversidad de los estudiantes. 
Barreyro (2008), observó que esta diversidad se debe al hecho de que ha habido una mayor participación del sector privado en la educación de los estudiantes durante los últimos diez años, lo que estimula la ampliación del acceso a la educación superior en Brasil.

Los cuestionarios fueron distribuidos a los estudiantes en una universidad privada de San Pablo, que imparte el curso de Tecnología en Seguridad de la Información. El número de estudiantes en las clases de encuestados fue de 110, aunque solo un 55\% (60 estudiantes) recibieron los cuestionarios, ya que la investigación se llevó a cabo al final del semestre.

Dos tipos de cuestionarios fueron administrados, uno de ellos enfocado en el conocimiento general de Gestión de Personas y su importancia (60 cuestionarios) y el otro solamente a los estudiantes que ya habían estudiado la asignatura(30 cuestionarios), el objetivo era identificar cuanto esta disciplina contribuye al desarrollo profesional. De los cuestionarios distribuidos, 93\% (56 cuestionarios) fueron respondidos. El perfil de los alumnos entrevistados y que se están graduando en Seguridad de la Información está compuesto, en su gran mayoría, de alumnos del sexo masculino (91\%) y con edades comprendidas entre los 18 y los 25 años (43\%), como se muestra en las figuras 1 y 2.

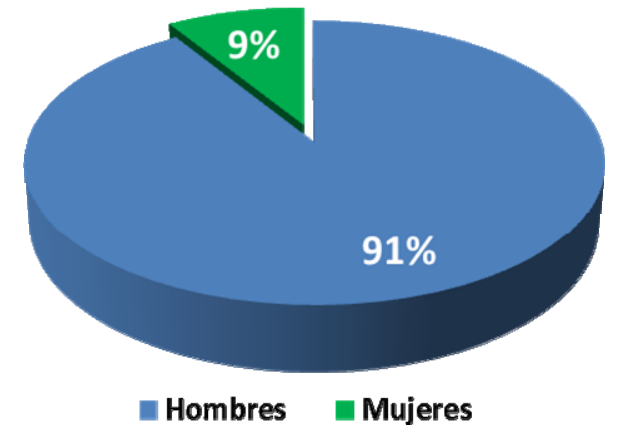

Fig. 1: distribución por sexo

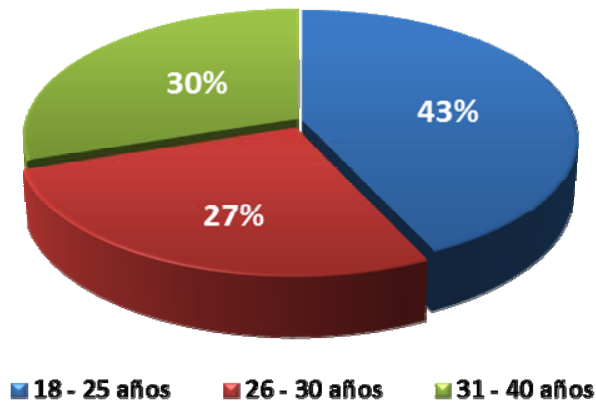

Fig. 2: distribución por edad

En cuanto a la ocupación profesional, de los 56 estudiantes entrevistados, 52 están empleados (93\%), tres son pasantes (5\%) y uno está desempleado (2\%), y el $62 \%$ están trabajando en el área de tecnología, como se muestra en las Figuras 3 y 4.

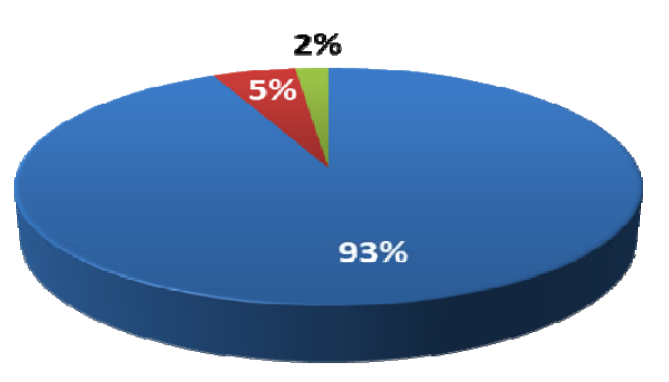

$\square$ Empleados 1 Pasantes $\square$ Sin empleo

Fig. 3: Ocupación

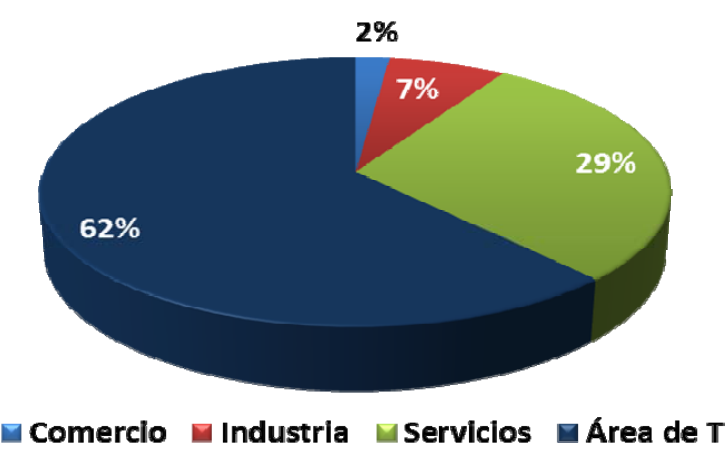

Fig. 4: Segmento de Trabajo

De los 55 estudiantes que trabajan, seis ya están trabajando en el área de Seguridad de la Información, dos de ellos tienen un papel de liderazgo. 52 de los 56 estudiantes expresaron su interés en mantener o convertirse en líderes en el futuro. Se obtuvo un porcentaje de $38 \%$ de los estudiantes que aún no están cursando la asignatura.

Nos dimos cuenta de que el $64 \%$ de los estudiantes, que ya ha estudiado Gestión de Personas, creen que la asignatura ha contribuido a su carrera profesional. Se observó que el $93 \%$ de los estudiantes creen que la disciplina ha colaborado para que sean buenos líderes desde su 
graduación, se destaca que el $7 \%$ que dijo que la asignatura le ha aportado beneficios, son los que no la había estudiado aún.

Hay una cierta expectativa de los estudiantes para aprovechar los conocimientos adquiridos durante el curso de graduación para obtener un puesto de liderazgo. Es interesante resaltar que el 23\% de los estudiantes entrevistados que ya habían estudiado Gestión de Personas respondió que aún no lo había hecho, lo que demuestra que algunos estudiantes tienen una visión distorsionada de la disciplina y/o no entendieron que ya la habían cursado.

Algunos estudiantes se quejaron de que el contenido del currículum no es aplicable en las empresas, debido a las políticas y culturas internas de las mismas, es por eso que no mostraban interés. Por otra parte, algunos alumnos señalaron que cambiaron su actitud y tuvieron éxito al hacerlo. Cuando se le preguntó acerca de la importancia de la disciplina de Gestión de Personas, el 7\% dijo que este tema no es importante. Algunos estudiantes reportaron haber hecho cursos en gestión de personas en las empresas donde trabajan o por cuenta propia (14\%).

De los estudiantes que ya la han estudiado, el 66\% respondió que la disciplina de Gestión de Personas, recibida en los cursos de graduación, han contribuido de alguna manera a mejorar su desempeño en las empresas donde trabajan. Algunos expresaron que hubo un cambio de comportamiento y la comprensión de la importancia de Gestión de Personas para el ejercicio de actividades profesionales relacionadas con la seguridad de la información. El veinte por ciento de los estudiantes creen que la que hay una carga horaria excesiva dedicada al tema, por otro lado, algunos creen que la carga horaria es pequeña para la importancia del tema.

Cuando se le preguntó sobre el contenido tratado en clase, el $20 \%$ de los estudiantes creen que no es compatible con la realidad en las empresas donde trabajan, sin embargo, las bases de este comentarios negativos son, de hecho, un punto positivo si tenemos en cuenta que el 100\% de quienes expresaron esto, dijo que la forma en que los equipos y sus líderes deberían actuar es siguiendo los conceptos tratados en la clase.

El muestreo se llevó a cabo con los estudiantes que ya han estudiado Gestión de Personas, se le distribuyeron 30 cuestionarios a este grupo y se observó un resultado significativo sobre cambio de comportamiento, después de asistir a la disciplina de Gestión de Personas, el 83\% de los estudiantes, expresaron que cambiaron su comportamiento y conducta en los grupos de trabajo. Aunque no desempeñaran un papel de liderazgo, hubo cambios positivos en su actitud, como se muestra en el tabla 1.

Tabla 1: Evolución de los conocimientos de los estudiantes en Gestión de Personas

\begin{tabular}{c|c}
\hline Evolución del conocimiento & Porcentual de los estudiantes \\
\hline sin cambios & $19 \%$ \\
De ningún para medio & $6 \%$ \\
De ningún para alto & $4 \%$ \\
De poco para medio & $21 \%$ \\
De poco para alto & $21 \%$ \\
De poco para muy alto & $6 \%$ \\
De medio para alto & $21 \%$ \\
De medio para muy alto & $2 \%$ \\
\hline
\end{tabular}

El Tabla 1 muestra el aumento del conocimiento de los estudiantes entrevistados sobre el tema de Gestión de Personas. El porcentaje de la evolución de escaso conocimiento para medio y alto, alcanza un total de $42 \%$, esta cifra muestra que el nivel de conocimiento ha tenido una evolución importante. Gran parte de los estudiantes (67\%) indicó que el tema del liderazgo y las habilidades interpersonales son los más importantes y relevantes de los tratados en la disciplina. 
Otra cuestión abordada en la investigación cualitativa, se aplica a la enseñanza interactiva y la disciplina en el aula cuando docencias estas temas en las aulas, donde podemos demostrar que el $90 \%$ de los estudiantes dijo que la forma lúdica y el uso de casos reales son muy útiles para comprender los problemas y temas tratados en clase.

A través de la encuesta de la gran mayoría de los estudiantes que participaron en el proyecto consideran que esta disciplina es importante para su carrera futura, a pesar de la resistencia inicial. Si bien realizados, la disciplina de Gestión de Personas se puede cambiar el comportamiento y la actitud de estos profesionales futuros.

Quedó claro a través de esta investigación que, para que los estudiantes absorban mejor conocimiento y se dediquen al aprendizaje, es necesario que todos los temas tratados en clase se les enseña a través de juegos y con la aplicación de estudios de caso reales. Para los estudiantes a los cuales esto aún no les sea útil, es evidente que pueden tener una idea de cómo el tema de la gestión de personal es importante, pero sin su aplicación adecuada, puede generar cierto escepticismo.

Según Tena (2010), cabe destacar que la comunicación entre profesor y estudiante adquiere una importancia relevante en el proceso de aprendizaje.

Se ha observado que la resistencia de los estudiantes se deshace cuando se dan cuenta que los temas tratados en el aula reflejan la realidad que viven en las empresas. El tema de liderazgo se entiende mejor entre los estudiantes que ya tienen una posición de liderazgo, los estudiantes que aún no ostentan estos cargos tienen otro punto de vista. Se observó que los estudiantes comenzaron a darse cuenta de la importancia del papel de líder y de cómo su enfoque pueden afectar los resultados.

\section{CONCLUSIONES}

Los resultados de esta encuesta indican que la disciplina Gestión de Personas, aunque en un principio no es tan importante para los estudiantes como las asignaturas técnicas, al final, se mostró que la misma fue efectiva para aumentar el interés de los alumnos, y por lo tanto, su importancia para mantener un estrecho diálogo con los estudiantes a fin de mantener el plan de estudios interesante y actualizado

Es importante destacar que en todos los grupos estudiados, se abordó el tema de la misma manera y los resultados fueron los mismos, esto demuestra que el tema Gestión de Personas se percibe de manera similar por los estudiantes, ya que la encuesta se llevó a cabo con estudiantes de diferentes campus. Con base en esta investigación se podría tener una percepción de que los estudiantes que han estudiado y los que no lo han hecho, tienen la misma opinión sobre el tema de la Gestión de Personas.

Con este estudio, podemos entender la importancia de la disciplina Gestión de Personas en la formación de esta profesión, ya que estos profesionales, durante sus carreras y actividades profesionales, utilizan los conceptos y las enseñanzas que tuvieron durante la graduación, y esto es además un factor diferenciador, ya que un profesional que tiene una visión técnica aliada con la visión de gestión tiene más oportunidades en el mercado laboral.

Durante esta investigación se hizo evidente que, si se administra bien, la disciplina de Gestión de Personas puede contribuir mucho a la formación de excelentes profesionales en el área de Seguridad de la Información, y los prepara para actuar como líderes y como miembros del equipo y mantener así la integridad y seguridad de lo más importante para el negocio: la información, la comunicación y el trabajo en equipo.

Con base en la información obtenida, se concluye que la disciplina Gestión de Personas, contribuye significativamente a las carreras los estudiantes de graduación en Seguridad de 
Tecnología de la Información y que también puede mejorar la manera de presentarle esta disciplina a los estudiantes que tienen perfiles técnicos.

La investigación examinada aquí puede servir de base para las universidades que deseen mejorar sus cursos de graduación, y así formar un profesional mejor preparado para entrar en un mercado de trabajo cada vez más exigente y competitivo.

El estudio tendrá su continuidad a través de un análisis más profundo del perfil de los profesores que enseñan la disciplina de Gestión de Personas en los cursos de Seguridad de Tecnología de la Información, analizando el impacto en la motivación de los estudiantes.

\section{AGRADECIMIENTOS}

Este trabajo fue financiado por la Universidad Nove de Julho. Estamos muy agradecidos a los revisores anónimos por sus importantes observaciones para mejorar la calidad del trabajo.

\section{REFERENCIAS}

4hd, $1^{\mathrm{a}}$ Pesquisa Nacional sobre Gestores de Help Desk, Service Desk e Suporte Técnico. Publicada em 24/05/2010, http://www.4hd.com.br (2010)

Allen, D. y Wilson, T. D., Information systems strategy formulation in higher education. In Understanding information policy: proceedings of a British Library funded Information Policy Unit Workshop, Cumberland Lodge, UK, (1997).

Araujo, L.C.G., estratégias e integração organizacional. São Paulo: Atlas, (2006).

Barreyro, G.B. Mapa do ensino superior privado. Brasília, DF: MEC/INEP, (2008).

Cano, C. y Hernández S. C. Gestión en Línea de las Evidencias de Aprendizaje en Educación Superior. Revista Formación Universitaria, vol.4 no.6 La Serena. (2011).

Chiavenato, I. Recursos Humanos - O capital humano nas organizações. 9a ed. Rio de Janeiro: Elsevier, (2009).

Dalmazo, L. "Ten tips for a better IT manager", ComputerWorld, (2007).

Dutra, J. S. Competências: conceitos e instrumentos para a gestão de pessoas na empresa moderna. São Paulo: Atlas, (2004).

Fleury, M. T. L. "Business strategies and skills training", Rio de Janeiro: Atlas, (2001).

Galve-Górriz, C., Castel A. G. Journal of theoretical and applied electronic commerce research (2010).

Longo, F." The time and place of the people", Fundap Magazine, (2009).

Marconi, M.A.; Lakatos, E.M. Técnicas de pesquisa: planejamento e execução de pesquisas, amostragens e técnicas de pesquisas, elaboração, análise e interpretação de dados. 6.ed. São Paulo: Atlas, (2007).

Rezende, D. A. "Software engineering and information systems", 2. ed. Rio de Janeiro: Brasport Multimedia Books Ltd., (2002).

Ruiz, M. "For excellence in technology", São Paulo: Brazilian Association of Software Companies, (2007). 
Security Review Journal, 10 th edition of the National Survey of Information Security of Software Companies, (2007).

Starec, C.; Gomes, E. B. P.; Chaves, J. B. L. Gestão Estratégica da Informação e Inteligência Competitiva. São Paulo: Saraiva, (2005).

Tena, M. Aprendizaje de la Competencia Creatividad e Innovación en el marco de una titulación adaptada al Espacio Europeo de Educación Superior. Formación Universitaria - Vol. 3 No 2 (2010). 